\title{
Reflets
}

Revue ontaroise d'intervention sociale et communautaire

\section{La participation populaire engagée et organisée : une pratique sociale à l'image des Acadiens}

\section{Claude Snow}

Volume 6, numéro 2, automne 2000

Problèmes sociaux en Ontario français

URI : https://id.erudit.org/iderudit/026321ar

DOI : https://doi.org/10.7202/026321ar

Aller au sommaire du numéro

Éditeur(s)

Reflets : Revue ontaroise d'intervention sociale et communautaire

ISSN

1203-4576 (imprimé)

1712-8498 (numérique)

Découvrir la revue

Citer cet article

Snow, C. (2000). La participation populaire engagée et organisée : une pratique sociale à l'image des Acadiens. Reflets, 6(2), 182-200.

https://doi.org/10.7202/026321ar

Tous droits réservés (C) Reflets : Revue ontaroise d'intervention sociale et communautaire, 2000
Ce document est protégé par la loi sur le droit d'auteur. L'utilisation des services d'Érudit (y compris la reproduction) est assujettie à sa politique d'utilisation que vous pouvez consulter en ligne.

https://apropos.erudit.org/fr/usagers/politique-dutilisation/ 


\title{
La participation populaire engagée et organisée $\square$ : une pratique sociale à l'image des Acadiens
}

\author{
Claude Snow \\ Travailleur social autonome
}

\section{Préambule}

Le travail que fait, dans le nord-est du Nouveau-Brunswick, le Comité des 12 pour la justice sociale, cadre bien dans l'optique du présent numéro de Reflets qui porte sur les problèmes sociaux. Après avoir brossé un tableau des principales caractéristiques de la Péninsule acadienne sur le plan socioéconomique, nous traiterons de la nature particulière du Comité des 12 et de l'apport de la profession du service social. Un regard sur les particularités des milieux ruraux et minoritaires et des défis qu'ils offrent aux intervenants permettra de conclure à la possibilité de résoudre les problèmes liés à la mobilisation, à l'engagement social et à l'organisation au sein des groupes populaires.

\section{La montée d'une nouvelle droite}

À prime abord, il parait impossible de faire obstacle à la marée montante au pays d'une nouvelle droite. Fort heureusement, il y a moyen de rester à flot au sein de ce brouhaha idéologique. La 
participation populaire engagée et organisée peut devenir pour certains une planche de salut à laquelle s'accrocher pour combattre le feu par le feu.

Les gouvernements, fédéral comme provinciaux, ont élaboré, on le sait, tout un arsenal démagogique basé sur l'idée que c'est à chacun, et non plus à l'État, de voir au bien commun, autrement dit, de travailler à renforcer sa communauté. Ce renforcement des capacités communautaires a ses vertus. Cependant, nous constatons que le revenu annuel de ceux qui prêchent le plus est de cinq à dix fois supérieur à celui de ceux qu'ils cherchent à convaincre.

Pendant qu'on prône l'urgence d'un nouveau type de société, l'État-Providence, lui, continue sa dégringolade. Il est difficile de croire qu'en si peu de temps, un aussi bon système de protection sociale ait pu être rayé de la carte. Il y a même une sorte d'amnésie collective qui facilite l'endoctrinement et qui permet d'oublier que la première fonction de l'État est de protéger ses citoyens.

Le mouvement néolibéral présente la société civile comme garante d'un monde plus gentil, où les politiques froides et rigides sont choses du passé. Ce que nous voyons en réalité, ce sont des pauvres qui ne peuvent plus compter sur l'État et qui doivent quêter du secours auprès d'organisations caritatives. Pourtant, comme le faisait remarquer un jour Ralph Nader, lorsque la justice prévaut, point n'est besoin de charité.

Les régions défavorisées se sentent trahies par le gouvernement fédéral qui a battu en retraite face à ses responsabilités en matière sociale. Là où l'économie est faible, il est évidemment impossible de réclamer du secteur non gouvernemental et de l'entreprise privée des investissements sociaux en remplacement de ceux du gouvernement.

Le système d'assistance sociale, jadis pierre angulaire du système de sécurité du revenu au pays, est en train de s'effriter, peu à peu remplacé par des programmes artificiels de formation et de création d'emploi. Depuis l'abolition du Régime canadien d'assistance publique au profit des transferts sociaux canadiens, les provinces sont libres de secourir à leur guise les personnes dans le besoin. Ce sont donc les plus vulnérables qui en paient la note. 
La réforme de l'assurance-chômage a elle aussi contribué à l'insécurité du revenu. Ceux qui dépendent d'emplois saisonniers se voient privés, durant les périodes de chômage forcé, de prestations qui leur reviennent en toute légitimité. En fait, les politiques de nivellement de l'assurance-chômage ne respectent pas les différences entre les divers types d'emploi. Elles portent atteinte aux travailleurs et aux travailleuses qui, non pas par choix mais en raison des conditions économiques, occupent des emplois saisonniers, temporaires, à la pige, à contrat ou à temps partiel.

\section{La Péninsule acadienne, ses forces et ses faiblesses}

Située au nord-est du Nouveau-Brunswick, la Péninsule acadienne s'étend sur une superficie d'environ 2000 kilomètres carrés.

À bien des égards, les problèmes sociaux du nord-est du N.-B. sont semblables à ceux qui se manifestent ailleurs au pays. Il convient cependant de souligner certaines particularités.

Le premier ministre Frank McKenna a déclaré en mai 1997 que la Péninsule acadienne a une "histoire de rébellion et de violence et que les gens ont l'habitude de recourir à la violence pour régler les différends ». Cette affirmation est loin de se vérifier dans les faits.

En réalité, ce qui caractérise les Acadiens, c'est plutôt leur grande résistance et leurs luttes pour le respect de leurs droits, tels les écoles homogènes, le maintien des écoles dans les petites communautés, les mouvements exclusivement francophones et le contrôle de la gestion scolaire. Ces luttes témoignent d'un peuple qui exerce des pressions dans le but de s'affirmer, et non en vue de commettre des actes d'agression.

Avec un visage culturel diversifié hérité d'ancêtres français, irlandais, écossais et amérindiens, la Pénincule acadienne compte environ 50000 francophones, ce qui représente $92 \%$ de la population. Les secteurs primaire et tertiaire, c'est-à-dire l'exploitation des ressources naturelles et les services publics, dominent 
l'économie. Plus du tiers des citoyens dépendent de transferts gouvernementaux pour vivre. De fait, une famille sur cinq est à faible revenu. La moitié des familles gagne moins de 30000 \$par année.

Le quart de la population est en chômage. Ceux qui travaillent ont des emplois précaires, c'est-à-dire saisonniers, occasionnels, sur appel ou de remplacement. Il y a peu d'emplois permanents, de qualité et bien payés et les nouveaux arrivants sur le marché du travail sont rémunérés au salaire minimum, sans grande protection sociale.

Selon les statistiques tirées du recensement de 1991, le problème fondamental dans la Péninsule acadienne ne se situe pas au niveau du taux d'activité $(76 \%$ de la population active est au travail), mais plutôt à celui du type d'activité rémunérée. En effet, $77 \%$ des personnes employées font du travail précaire et $23 \%$ seulement travaillent à temps plein toute l'année contre les $46 \%$ qui occupent un emploi stable à l'échelle de la province.

Plusieurs indicateurs témoignent d'un affaiblissement de l'économie, à partir de l'exode des jeunes ménages, de la vente forcée de centaines de maisons, de la perte d'emplois permanents bien rémunérés, substitués par des emplois rémunérés au salaire minimum, jusqu'à l'augmentation du nombre de faillites personnelles.

Sur le plan social, le tableau n'est guère plus reluisant. On note une augmentation du nombre d'enfants placés sous la protection de l'État, un taux de suicide chez les jeunes de 20 à 24 ans de $86 \%$ plus élevé que la moyenne canadienne et un taux de décrochage scolaire (29\% chez les élèves de 13 à 15 ans) de beaucoup supérieur à la moyenne provinciale.

Les statistiques du recensement de 1996 ont révélé que la Péninsule acadienne se situe au deuxième rang, après le comté de St-Jean, en ce qui a trait au pourcentage de familles monoparentales. Par ailleurs, en 1997, selon le ministère de la Santé et des Services communautaires, $40 \%$ des nouveau-nés dans la Péninsule acadienne sont à risque, leur poids à la naissance étant en deça de la norme. 
Au cours des dernières années, le mouvement vers la mondialisation, l'automatisation, la dévolution, la privatisation et la soustraitance a frappé dur la Péninsule acadienne où la raréfaction des ressources naturelles se fait sentir.

Les politiques centralisatrices et la perte de pouvoir local qui s'ensuivit, la loi provinciale antidéficit, la perte de services statutaires devenus des privilèges, les coupures au niveau des services de soutien et le processus bien arrêté de dévitaliser les régions rurales au profit du triangle doré du sud, c'est-à-dire Moncton, St-Jean et Fredericton, ont sonné le glas du filet de sécurité sociale qui n’a cessé depuis de s'effriter.

Il y aurait de quoi s'alarmer et la situation serait désespérante n'eut été l'apparition d'une nouvelle combativité chez les citoyens en quête de gouvernements plus humains et plus sensibles à leurs besoins.

Parce qu'ils voulaient s'opposer au régime opprimant de Chrétien et de McKenna, une douzaine de citoyens de la Péninsule acadienne ont mis sur pied un comité de citoyens, le Comité des 12 pour la justice sociale, dont le mandat est de lutter pour la préservation de la dignité et pour la défense des droits sociaux. Le Comité aide les citoyens à faible revenu à prendre la place qui leur revient et à marcher la tête haute, dans l'honneur et dans la dignité.

Le Comité des 12 représente toutes les couches de la société, notamment, les personnes âgées, les membres des syndicats, les bénéficiaires d'aide au revenu et de services de santé mentale, les bénévoles, les professionnels, le clergé, les enseignants et enseignantes et le monde ouvrier. Certains membres sont eux-mêmes des bénéficiaires de l'aide au revenu ou à la santé mentale, alors que d'autres sont des membres de la communauté militant pour le respect des personnes dans le besoin.

Ainsi,le Comité des 12 est un organisme non gouvernemental, apolitique, de sensibilisation et de revendication qui traite de plusieurs dossiers. Ses interventions consistent la plupart du temps à écrire des lettres pour résoudre les problèmes individuels; il fait également de la médiation, de la négociation et diverses 
représentations par le biais de mémoires, de causeries et d'entrevues radiophoniques ou télédiffusées. Il intervient autant aux plans de l'aide au revenu, de l'habitation, de l'emploi et de l'aide juridique qu'à ceux des autres services publics où les citoyens se butent à une bureaucratie oppressante et déshumanisante.

Par exemple, le Comité a déjà dénoncé les tactiques intimidantes des enquêteurs de l'aide au revenu qui soupçonnaient toujours les bénéficiaires d'être des abuseurs. Les personnes à faible revenu se sentaient constamment menacées par l'insécurité financière, les avertissements, les fouilles, les saisies, les enquêtes et diverses autres formes de harcèlement. Grâce aux nombreuses pressions exercées par le Comité, ces tactiques ont pratiquement disparu.

Le ministère du Développement des ressources humaines du Nouveau-Brunswick a également accepté de verser des prestations plus élevées aux personnes qui ont des problèmes autres que physiques, sans qu'elles aient à soumettre de rapports médicaux, si un professionnel de la santé mentale ou des services sociaux atteste d'un handicap permanent qui les empêche de travailler. Le ministère a aussi adopté un système préconisé par le Comité des 12 qui consiste à fournir aux bénéficiaires de l'aide au revenu leur dossier d'admissibilité. De plus, il a accepté de décentraliser le système d'appel et de nommer un tribunal d'appel dans chaque région de la province. Enfin, il a assoupli sa politique concernant la mise à terme de l'assistance et il donne maintenant un préavis d'un mois avant d'y mettre fin.

Face à la détresse de tant de personnes démunies, affaiblies et vulnérables, le Comité des 12 a proposé d'aider ces dernières à s'associer pour défendre et promouvoir leurs intérêts. Il leur vient en aide par le dépannage individuel, la défense collective et la promotion des valeurs sociales.

Le Comité des 12 fonctionne depuis maintenant sept ans et il traite une centaine de dossiers par année. Il vise l'élimination des inégalités sociales et l'amélioration de la condition humaine. Prenant le gouvernement au mot, il a invité les citoyens à faire une contribution sociale. La sienne consiste à enseigner aux 
citoyens à s'affirmer collectivement. Autrement dit, il n'était pas possible de lutter contre un courant aussi vaste que l'idéologie néolibérale, mais le Comité des 12 a réussi à récupérer à son avantage le discours politique.

Évidemment, la perception gouvernementale du partenariat n'est pas celle des membres du Comité. Pour ces derniers, le partenariat consiste à aider les citoyens à militer, à s'affirmer, à revendiquer, à faire valoir leurs droits et à défendre leur dignité, et non simplement à préparer des boîtes de nourriture pour ceux qui ont faim. En d'autres mots, ils montrent aux gens à pêcher, plutôt que de leur donner du poisson.

Le Comité n'est pas très tendre envers les fonctionnaires et les politiciens. Un comité de citoyens n'est pas une confrérie de dames patronnesses. Parce qu'il se livre sans répit au talonnage bureaucratique, il est devenu « la mouche sur le coche » pour le gouvernement, comme l'a justement fait remarquer quelqu'un.

Le Comité ne sera pas satisfait tant que les politiques publiques ne seront pas ajustées aux réalités socioéconomiques de la Péninsule acadienne.

Il devient de plus en plus évident pour le Comité que l'uniformité ne rend pas justice aux différences régionales. L'uniformité est égale, mais inéquitable, parce qu'elle ne tient pas compte de la diversité régionale. Pour répondre aux besoins des régions, il faut que les politiques prévoient des mesures d'exception là où les conditions sont différentes.

Le Comité assume une fonction de vigilance sociale qui s'étale sur trois fronts: la protection et l'aide à l'autonomie, la défense des droits et la revendication, la promotion de la sécurité et de la justice sociale.

Les membres croient que les citoyens à faible revenu ont droit à un minimum vital et à des conditions de vie convenables, comme c'est le cas pour les autres citoyens. Pour les aider à s'en prévaloir, ils leur enseignent à organiser des pétitions, des campagnes de sensibilisation et à établir des stratégies politiques et médiatiques. Ils les aident aussi à se défendre face à la bureaucratie rigide et aveugle. 
Sur le plan de la défense sociale, le Comité agit comme une sentinelle des droits sociaux. Il parle et écrit au nom de ceux qui ne peuvent livrer leur propre combat et il dénonce les atteintes aux droits et les effets pervers de la répression.

Le Comité défend aussi le droit des citoyens à la protection sociale et à l'équité et il appuie toute bonne initiative de la part du gouvernement à cet égard. Il ne manque pas, cependant, de réprouver toutes celles qui vont en sens contraire et il se fait un devoir de rappeler continuellement au gouvernement son mandat social.

Évidemment, tout le travail du Comité se fait bénévolement car, avec le recul du développement social, le gouvernement a retiré son appui aux organismes communautaires.

\section{L'apport de la profession du travail social}

Le service social est particulièrement sensible aux rapports de force, et par conséquent, il a beaucoup à offrir à ceux qui se dirigent vers l'action communautaire, la défense des droits et la militance en général. Le modèle de participation engagée et organisée que le Comité des 12 a adopté s'inspire donc, en grande partie, de la pratique du travail social.

Les concepts de mobilisation, de participation, de renforcement et d'entraide ne sont pas étrangers aux valeurs professionnelles du travail social mais, parce qu'ils se vendaient bien, ils ont été usurpés et utilisés à des fins politiques et économiques plutôt que sociales. En conséquence, le gouvernement s'éloigne de plus en plus d'une approche intégrale où les dimensions économique et sociale se complètent.

En puisant dans le riche répertoire de possibilités que nous offre le travail social collectif, il est possible de renverser la vapeur, ou du moins de créer quelques soupapes. C'est donc dire que cette bonne vieille méthode du travail social collectif peut encore rendre de fiers services aux citoyens. 
Les travailleurs sociaux croient que l'engagement social est une force latente qui peut être réveillée et exploitée, en autant que les citoyens sachent comment s'en servir pour promouvoir leurs besoins et leurs intérêts. Il suffit de miser sur la débrouillardise et sur le savoir-faire des citoyens et de les amener à inventer des projets à leur image et à leur goût.

Les travailleurs sociaux aident les gens à renforcer leur combativité par divers moyens dont l'action communautaire, l'animation sociale, le travail de groupe, la défense des droits collectifs et individuels et la vigilance sociale. Ce sont là des approches qui sont propres au travail social.

Le travailleur social joue au sein des groupes, le rôle d'un défenseur et d'un conseiller. Souvent, c'est lui qui donne "l'âme " au groupe, lequel ressemble à un organisme vivant devant évoluer de façon organisée. Dans sa militance le groupe a donc besoin d'outils, d'une méthode et de techniques et bien souvent, d'un appui professionnel.

Souvent dans les groupes, des personnes ont des problèmes personnels importants. Le travailleur social doit, au besoin, faire de la consultation individuelle et du dépannage. Il offre surtout des sessions de formation populaire et il fournit des avis sociaux au niveau des diverses tâches que le groupe doit accomplir, comme par exemple, le réseautage et la correspondance.

Le travailleur social agit aussi comme un catalyseur. Il apporte au groupe une nouvelle vision. Puisqu'il est de l'extérieur du groupe, il jette un éclairage différent. "On ne peut pas résoudre un problème avec la même pensée que celle qui l'a créé ", disait Albert Einstein, une constatation qui s'applique bien dans le domaine de la défense collective.

Quand les professionnels prennent trop de place au sein des groupes, leurs membres sont inhibés dans leur créativité. Par contre, quand la participation professionnelle en est une d'appui, elle débouche alors sur la liberté et devient une force créative offrant une lueur d'espoir aux individus. 


\section{Les milieux ruraux et minoritaires et les valeurs qui leur sont propres}

Il aurait été impensable pour le Comité des 12 de promouvoir la participation populaire en ignorant la lutte sur plusieurs fronts que mènent en ce moment les milieux ruraux et minoritaires. En fait, les membres du Comité sont eux-mêmes partie intégrante de ces luttes collectives qui visent à assurer la survie culturelle, à bâtir la communauté autour de l'école, à éveiller le sentiment d'appartenance à la collectivité et à renforcer l'identité et l'esprit de solidarité. Comme le constatait Gian Giacomo Zucchi, la lutte perpétuelle du minoritaire est de poursuivre son existence, sa langue, son identité et son estime.

Les valeurs collectives et les traits culturels prennent une importance capitale chez les communautés minoritaires et on doit en tenir compte. Alors que les groupes majoritaires ont plutôt tendance à valoriser les intérêts individuels, les groupes minoritaires favorisent les intérêts communautaires. On note par exemple chez les Acadiens l'importance de la vie associative pour l'identité et la survie culturelle, la lutte pour obtenir des structures homogènes et la priorisation de la vie sociale par rapport à la vie économique, ce qui se voit moins chez leurs concitoyens anglophones. Les populations disséminées des milieux ruraux sont plus affectées que les autres par les coupures au niveau des services sociaux, d'où l'obligation de toujours tenir compte des diverses composantes des problèmes sur les plans socioéconomique, démographique, géographique et culturel.

Là où le niveau socioéconomique et la densité de la population sont faibles, il est préférable de s'éloigner des modèles qui fonctionnent bien dans les centres urbains pour en créer de nouveaux, plus compatibles avec les réalités rurales.

Les milieux ruraux ont leurs propres caractéristiques. Le mode de vie y est différent, tout comme l'est la façon de régler les problèmes humains. Dans les petites communautés, il y a une façon différente d'aider les gens en difficulté. Les gens se portent 
plus au secours des autres. Ils s'engagent de façon personnelle et informelle. Le problème d'un citoyen devient le problème de tous. Les conflits sont réglés d'une manière plus directe. Les gens ont le sens du regroupement. Quand leur communauté est menacée, ils se réunissent et luttent pour leur survie.

Tous les milieux ruraux ont de la difficulté à accéder aux services et, de surcroit, les milieux francophones doivent faire face à des problèmes d'ordre linguistique. De plus, chez les francophones, les relations sociales et l'identité communautaire priment les aspects économiques et pragmatiques. Par ailleurs, certains principes sont chers aux Acadiens, dont la dualité administrative, l'égalité des chances et l'autorité locale. Cela aussi doit être pris en compte.

\section{L'engagement comme point de départ}

Le nombre d'embûches à surmonter dans le travail collectif oblige les participants à faire de la défense des droits sociaux une question d'honneur, de dignité et de fierté. Il s'agit, en somme, d'un état d'esprit à développer. Les personnes qui s'engagent ont habituellement une conscience sociale et elles sont sensibles aux questions sociales. Elles travaillent ainsi à l'amélioration de la qualité de vie de leurs concitoyens.

La démocratie fonctionne mieux quand les différences idéologiques s'affrontent. Comme l'affirmait Aristote, l'équité est mieux servie quand tous les citoyens participent à leur gouvernement. En fait, le pire ennemi du progrès social, c'est la satisfaction. Or, aucun progrès social n'est possible sans changement, résultant de l'influence des groupes de pression. Pour qu'il y ait un progrès social, il faut que des gens s'engagent à améliorer les conditions sociales, ce qui n'est pas possible quand ils en sont satisfaits.

L'engagement organisé est l'opposé de l'indifférence et de la démoralisation collective. Ceux qui s'engagent dans la participation 
populaire le font parce qu'ils préferent combattre plutôt que de se laisser abattre. Ils croient aussi qu'ensemble, ils peuvent se donner une force qu'ils n'ont pas individuellement.

Sortir de son isolement, se regrouper et chercher ensemble des solutions sont aussi des expériences enrichissantes. La créativité est en effet la ressource la plus importante et la moins chère dont disposent les groupes. Même quand d'autres ressources font cruellement défaut, il y a toujours moyen de compter sur la force de l'imagination pour s'en sortir. La créativité est utilisée pour élaborer des stratégies, et plus les stratégies sont futées, plus elles sont efficaces.

Certains sont à la recherche de "perles rares " pour former des groupes de citoyens, alors que d'autres soutiennent que la bonne volonté suffit. La vérité se situe à mi-chemin. D'abord il faut se rendre à l'évidence qu'il y a beaucoup plus de gens ordinaires qu'il y a de perles rares. Comme le faisait si bien observer un auteur français, Dieu a dû aimer le commun des mortels pour en avoir créé autant! Par contre, la bonne volonté sans les autres ingrédients conduit généralement à un cul de sac, puis à l'échec. Ce qu'il faut se dire en partant, c'est que les gens ordinaires comptent plein de têtes savantes et qu'il est possible, avec un peu d'orientation, de les amener à participer de façon engagée et organisée.

Il est possible pour les membres d'un groupe de lutter ensemble et de réaliser des petites victoires, à condition toutefois qu'ils soient prêts à faire équipe, à mettre leurs talents à la disposition du groupe et puis, à collaborer en poursuivant un même but, ce qui est plus simple à dire qu'à faire. Il faut une certaine abnégation de la part des membres pour nourrir un projet commun, plutôt que de vouloir poursuivre des intérêts personnels. En fait, les intérêts doivent être réconciliables et il faut que les individus veuillent travailler dans la complicité à réaliser le même rêve.

La mobilisation des citoyens autour d'un projet commun consiste à rassembler les forces pour se donner un pouvoir d'influence. Le groupe doit toujours se rappeler que ce qu'il représente est plus important que ce qu'il est et il doit être persuadé 
que les pressions sociales qu'il exerce ont une influence déterminante sur la prise de décision, même si les résultats ne sont pas toujours tangibles. Un groupe de gens qui réalise qu'il peut faire bouger les choses, et qui s'organise, représente une force incroyable.

La prise de décision peut être comparée à une grande couverture que chacun tire de son côté. L'enjeu consiste à amener la partie adverse à déroger un peu de sa position, ce qui ne se fait pas sans déranger l'ordre établi. Or, il faut que les groupes populaires se prémunissent contre la critique négative. Ils ne feront pas long feu s'ils attendent de faire l'unanimité autour d'eux avant de passer à l'action. En fait, ils doivent devenir imperméables à certaines critiques pour pouvoir poursuivre leur lutte. En nageant dans l'ambivalence et dans la neutralité, ils risquent de rester au beau fixe.

\section{L'organisation, la clé du savoir-faire}

Après l'engagement, l'organisation est l'arme la plus redoutable dont disposent les groupes populaires, mais encore faut-il qu'ils sachent comment y parvenir!

Bien entendu, les groupes populaires ne disposent pas des mêmes moyens que les instances décisionnelles. Alors que ces dernières ont l'autorité et les ressources nécessaires pour mousser leur message et financer leur action, les groupes populaires doivent recourir au pouvoir d'influence, aux stratégies d'intervention, aux médias et à la participation bénévole. Cependant, il ne faut pas minimiser l'impact de ces moyens car, bien utilisés, ils constituent une force de frappe appréciable.

La règle d'or de la participation organisée est le "9-1-1 Solidarité ", c'est-à-dire 9 personnes dont les compétences se complètent +1 cause +1 plan. Bien des animateurs sociaux ont longtemps pensé qu'il suffisait de trois personnes pour former un groupe; maintenant, à la lumière des nouvelles connaissances, ils préconisent plutôt la formation de groupes plus nombreux qui se complètent. 
Il n'y a rien comme une variété d'aptitudes au sein d'un groupe, qui peuvent se fondre pour constituer une force. Quand le groupe peut compter sur une personne qui connaît les procédures d'assemblées, une qui connait les rouages de la fonction publique, une qui est bonne à inventer des stratégies, une autre qui est à l'aise avec les médias, une qui prélève des fonds, une qui peut écrire et préparer des budgets, une qui sait remplir des demandes de projets, une autre qui maîtrise les aspects techniques et les ordinateurs et une dernière qui connait bien les personnesclés de la communauté, il possède une banque de ressources qui peut l'amener très loin.

Il n'est pas toujours possible d'avoir autant de compétences autour d'une table. Ce qui importe, c'est d'avoir au moins une personne capable de proposer des idées nouvelles, une autre habile en rédaction et en communication et une autre qui peut coordonner et faire la mise en oeuvre des décisions.

Une règle qui facilite beaucoup le travail de groupe est celle de la souplesse. Elle permet au groupe d'accomplir le plus possible avec le moins d'effort possible, dans un fonctionnement efficace, dépourvu de perte de temps. Personne ne s'intéresse à des réunions interminables ou à la rédaction de longs procès-verbaux.

Une autre règle est celle de l'acrobatie. Elle consiste à inventer de nouvelles formules qui se situent entre l'autocratie et la démocratie. Cette dernière a fait sa marque, bien entendu. Mais elle coûte cher et ce ne sont pas tous les groupes qui sont capables de s'en prévaloir. Les délais prescrits, le quorum, les propositions relatives aux questions préalables, etc. sont des procédures lourdes et encombrantes qui deviennent un carcan et font fuir des personnes qui seraient normalement intéressées à se joindre au groupe. Celui-ci doit chercher à inventer des formules qui visent à alléger et à faciliter son travail : confier, par exemple, à un membre la charge de faire avancer un dossier, pour ensuite faire des recommandations au groupe.

Le groupe doit apprendre à exercer son pouvoir d'influence et à poser les bonnes questions. En fait, il n'est pas nécessaire de 
trouver des réponses aux problèmes (quoique cela puisse faire partie de sa stratégie de collaboration). Il suffit de savoir quelles questions poser, à qui, quand et comment.

Il doit apprendre à utiliser le pouvoir des mots et le meilleur moyen de le faire c'est en écrivant des rapports, des mémoires et des comptes rendus et surtout, des lettres. Écrire une lettre précise, concise et percutante, où un problème est exposé en quatre ou cinq paragraphes, est un art qui ne fait pas appel à des ressources financières, mais à des capacités de synthèse et d'expression.

Le groupe doit aussi apprendre à se faire des alliés pour convertir le plus de gens possible à sa cause. Cela implique généralement une bonne utilisation des médias. Les rapports avec ces derniers sont le talon d'Achille de bien des groupes qui se sentent soit opprimés, incompris ou laissés pour compte par les journalistes, ne sachant pas comment intervenir auprès d'eux. Or, à moins que le groupe sache comment agir sur l'opinion publique en médiatisant ce qu'il fait, son message ne passe pas, peu importe sa valeur intrinsèque.

Les groupes populaires ne doivent jamais oublier que la presse est le tribunal du peuple et que c'est le seul où il n'y a pas de frais d'avocats à payer. Le groupe doit sans cesse revenir sur le même message apprêté de diverses manières. Il ne doit pas avoir peur de dire et de redire ce qu'il croit, car le dire une seule fois ne suffit pas. Il doit aussi se doter d'un système qui lui permet de traiter, d'enregistrer et de classer l'information obtenue, autrement, il s'épuise à courir après celle-ci.

Enfin, le groupe doit apprendre à revendiquer rationnellement. Cela veut dire réclamer ses droits avec insistance, oui, mais de façon civile et légitime, en adoptant des stratégies et des tactiques qui suivent une démarche logique. Pour être efficace, l'action sociale doit être engagée et organisée, rappelons-le, et cela ne laisse aucune place à l'improvisation. 


\section{L'invention de stratégies, la créativité au service de la justice}

Une fois qu'un groupe populaire est mobilisé, engagé et organisé, il ne lui reste qu'à inventer des stratégies pour obtenir ce qu'il veut. L'invention de stratégies fait toute la différence entre un groupe moribond et un groupe vivant. C'est avec l'imagination que le groupe réussit à élaborer des tactiques pour faire plier l'autre et à penser à des moyens d'action imprévus.

La revendication, à elle seule, n'est pas une bonne stratégie. Il faut aussi une part de collaboration au moment opportun. C'est ce qu'on appelle la stratégie du " géant gentil ", un bon dosage de confrontation et de collaboration, comme par exemple, exiger que telle mesure ne soit pas adoptée tout en proposant des solutions de rechange.

Le groupe doit mettre au point des recettes qui lui permettent de " prendre du poids ", c'est-à-dire de bâtir du capital et de devenir influent. Il construit peu à peu un répertoire où aller puiser de façon à ne pas avoir toujours à recommencer.

Les groupes n'ont pas les moyens d'avoir des stratégies coûteuses. De toute façon, ce ne sont pas les plus musclées qui comptent, mais les plus impressionnantes. En plus d'élaborer des stratégies, le groupe peut utiliser des tactiques qui lui permettent de durcir sa position ou de faire fléchir un tenant du pouvoir trop sensible à son goût à des intérêts opposés.

Une tactique, par exemple, consiste à plaider, à tenter de persuader l'autre de dire comme soi. Une autre consiste à résister, à ne faire aucun compromis, à s'opposer fermement, mais respectueusement. La résistance tenace est une arme efficace que le groupe peut employer à bon escient. Une troisième tactique consiste à exiger, c'est-à-dire à forcer l'autre à changer d'idée, ce qui n'est pas facile. Il faut que le groupe soit sûr de l'appui de ses membres avant d'utiliser cette tactique, car la moindre défaillance l'affaiblit considérablement. Enfin, un groupe peut décider de 
simplement céder. C'est un bon moyen de montrer qu'il est de bonne foi, patient et flexible, à condition de ne pas le faire en se sentant en position de faiblesse. Un groupe qui sait mettre de l'eau dans son vin au moment opportun peut s'attendre ensuite à la même chose de son adversaire.

Pour atteindre ses buts, le groupe doit essentiellement avoir trois types d'acquis: un plan, c'est-à-dire une façon de s'organiser pour obtenir ce qu'il veut; des ressources, c'est-à-dire de l'information, des talents, de l'appui et de l'audace, c'est-à-dire le courage de foncer et d'aller jusqu'au bout.

Au-delà de tous ces ingrédients, il faut surtout une cause à laquelle s'identifier et s'engager tout au long de son parcours. Avec une cause précise, la route est pavée. Sinon, le groupe ne réussit pas à convaincre qui que ce soit.

Le groupe doit aussi pouvoir compter sur une âme dirigeante qui l'amène à décider de l'action à entreprendre, de la personne à contacter ou à influencer, du lieu ou du niveau où s'adresser et des moyens à prendre tels qu'écrire des lettres, faire signer une pétition, etc. Le leadership peut être assigné à une personne ou varier d'un individu à l'autre, selon les besoins et les intérêts du moment. En bref, l'action du groupe se résume à trois verbes : voir, dire et faire. Il faut que le groupe se renseigne, qu'il dise aux autres ce qu'il essaie de faire et qu'il fasse ce qui s'impose.

\section{Le désir d'essaimer pour promouvoir la participation populaire}

Au départ, le Comité des 12 souhaitait que d'autres comités comme le sien voient le jour, comme autant de cellules vivantes faisant partie d'un même mouvement de défense des droits sociaux. Quoique cela ne se soit pas matérialisé, il y a lieu de croire que la défense collective gagne du terrain chez les gens, à en juger par la couverture médiatique gratuite dont le Comité bénéficie et par les nombreux témoignages d'appui qu'il reçoit de toutes parts. 
Ce regroupement fait mentir en quelque sorte l'adage que les pauvres sont invisibles alors que les riches sont intouchables et qu'il n'y a rien à faire puisque les consciences sont endormies. Grâce à ses nombreuses interventions, le Comité rappelle sans cesse la réalité quotidienne des personnes à faible revenu, ce qui n'est pas peu dire. Lors des dernières élections provinciales, le parti au pouvoir s'est fait battre à plate couture et il se peut que, jusqu'à un certain point, le Comité y ait été pour quelque chose.

Il est arrivé que les choses n'aient pas changé malgré l'acharnement du Comité des 12 à faire abolir ou modifier des politiques jugées disgracieuses pour la dignité humaine en plus d'être une atteinte aux droits et libertés. Mais la situation aurait sans doute été pire si les groupes de défense des droits n'avaient pas exercé des pressions continues.

Il y a encore beaucoup de pain sur la planche. Le barème de l'aide au revenu n'a pas augmenté au Nouveau-Brunswick depuis avril 1997 et il se situe parmi les plus bas au pays. Le Comité continue d'implorer le gouvernement de faire le rattrapage qui s'impose, puis de réviser les taux à chaque année. D'autres politiques, comme le partage du logement et des revenus quand il y a des enfants adultes au foyer et l'assistance à ceux qui attendent le chômage, constituent des chevaux de bataille importants pour le Comité qui est persuadé que le gouvernement doit faire mieux.

Le Comité des 12, comme tant d'autres, est un groupe en quête d'une plus grande justice sociale. Bien que limité dans son action faute d'un financement de base, il a l'intention de continuer ses activités tant que les deux paliers de gouvernement ne se montreront pas plus redevables en ce qui touche le respect des droits sociaux et économiques des citoyens.

Le Comité des 12 croit que son message, basé sur la défense des valeurs communautaires, de l'équité et des réformes sociales, est important et il veut le véhiculer le plus loin et le plus fort possible. Il sait qu'il est dans la mare le caillou qui fait jaillir des ondes autour de lui.

De façon lapidaire, disons que la raison d'être de l'entraide engagée et organisée c'est, ni plus ni moins, de se mêler des affaires 
des autres. À l'instar de ce que disait un jour un intervenant de l'Accueil Bonneau de Montréal, tout citoyen devrait dire à l'égard de ses compatriotes : «Ce n'est pas vraiment de ma faute s'il y en a qui ont faim, mais ça le deviendra si je ne fais rien". 